\title{
Conhecimento do cuidador acerca do banho no leito em uma instituição de longa permanência para idosos
}

\author{
Knowledge of the caregiver on bathing in bed in a long-term institution for the elderly \\ Conocimiento del cuidador sobre el baño en el lecho em centros de atención a largo plazo para \\ ancianos
}

\section{Resumo}

Este estudo objetivou descrever o conhecimento dos cuidadores, quanto a realização do banho no leito em uma Instituição de Longa Permanência para Idosos. Caracterizou-se como um estudo descritivo exploratório que foi realizado, no ano de 2017, em um município cearense. Os dados foram obtidos por meio de entrevista semiestruturada e interpretados conforme análise de conteúdo. Os resultados foram apresentados e discutidos através de três categorias que se originaram conforme as respostas dos participantes, a saber: 1 . O conhecimento científico como base para realização do trabalho do cuidador; 2. A técnica de banho no leito e materiais utilizados pelos cuidadores; 3. Banho proporcionando conforto e bem estar ao cliente. A partir dos resultados encontrados pode-se concluir que os cuidadores dominam parte considerável da técnica do banho no leito, entretanto alguns aspectos necessários não foram comtemplados por eles. Esses aspectos não abordados pelos cuidadores, quando aliados a problemas institucionais, como a carência de materias para higienização, prejudicam a qualidade do procedimento.

Palavras-chave: Envelhecimento; Cuidadores; Banhos.

\section{Abstract}

The main purpose of this study was to describe the caregivers' knowledge regarding the performance of bed bathing in a Long-term Institution for the Elderly. It was characterized as a descriptive exploratory study that was conducted in 2017, in a municipality of Ceará. The data were obtained through semi-structured interviews and interpreted according to content analysis. The results were presented and discussed through three categories that originated according to the responses of the participants, namely: 1. Scientific knowledge as the basis for carrying out the work of the caregiver; 2. The bedside bathing technique and materials used by caregivers; 3 . Bath providing comfort and well-being to the client.From the results found it can be concluded that the caregivers dominate considerable part of the technique of bedside bathing, however, some necessary aspects were not covered by them. These aspects not 
addressed by caregivers, when allied to institutional problems such as lack of materials for hygienization harm the quality of the procedure.

Keywords: Aging; Caregivers; Baths.

\section{Resumen}

Este estudio tuvo como objetivo describir los conocimientos de los cuidadores, en cuanto a la realización del baño en cama en una Institución de Atención de Larga Duración para el Adulto Mayor. Se caracterizó como un estudio exploratorio descriptivo que se realizó, en 2017, en un municipio de Ceará. Los datos se obtuvieron mediante entrevistas semiestructuradas y se interpretaron de acuerdo con el análisis de contenido. Los resultados fueron presentados y discutidos a través de tres categorías que se originaron de acuerdo a las respuestas de los participantes, a saber: 1. El conocimiento científico como base para el desempeño del trabajo del cuidador; 2 . La técnica de baño en cama y los materiales utilizados por los cuidadores; 3 . Baño que proporcione comodidad y bienestar al cliente. De los resultados encontrados se puede concluir que los cuidadores dominan una parte considerable de la técnica del baño en cama, sin embargo algunos aspectos necesarios no fueron abordados por ellos. Estos aspectos no abordados por los cuidadores, cuando se combinan con problemas institucionales, como la falta de materiales para la higiene, perjudican la calidad del procedimiento.

Palabras clave: Envejecimiento; Cuidadores; Baños.

\section{Introdução}

O envelhecimento da população é um fenômeno global em ascendência. Segundo a Organização das Nações Unidas (ONU) a quantidade de pessoas com idade acima de 60 anos cresce disparada em relação a outros grupos etários, estima-se que a taxa de crescimento da população idosa mundial seja de 3\% ao ano. A projeção de idosos para o ano de 2050 é de 2,1 bilhões e após 50 anos, em 2100, o total de idosos pode aumentar em um bilhão a mais.

Pode-se conceituar o envelhecimento como um processo decorrente do ciclo de vida do ser humano, que se manifesta através de aspectos fisiológicos e biopsicossociais. Esse processo é caracterizado pela diminuição da reserva funcional, onde se é nomeado por senescência, com isso o decorrer da idade pode ser seguido por doenças crônico-degenerativas, deteriorização das funções e debilidades que acarretam perda ou diminuição da capacidade funcional, sendo definido como senilidade (Brasil, 2006).

A pessoa idosa demanda de atenção direcionada, necessitando de cuidados para realizar atividades diárias e cuidados em geral, o que torna necessária a presença de um cuidador para prestar assistência a esse individuo. Com base em suas muitas demandas alguns idosos não são tratados de uma maneira conveniente, então a maioria de seus familiares optam por abrigá-los em instituições de longa permanência (Poltronieri et al., 2019; Paula et al., 2017a).

A Instituição de Longa Permanência para Idosos (ILPI) foi designada para romper com ideologia negativa provocada pelo Asilo, que era, na maioria das vezes, visto como um local onde o idoso é abandonado e vive em situação de descaso (Costa \& Mercadante, 2013). A ILPI é definida como um espaço habitacional coletivo que abriga e oferta cuidados básicos como higiene, saúde, alimentação e promove bem-estar a pessoas idosas que no Brasil são os indivíduos com idade igual ou superior a 60 anos. Essas entidades atendem os idosos independentemente de condições financeiras, familiares ou situação de vulnerabilidade (Camarano \& Kanso, 2010)

Nessas instituições o trabalho de assistência ao idoso é realizado em grande parte pelo cuidador, pois de acordo com Camarano e Kanso (2010) as ILPI apesar de ofertarem atendimento médico e medicações não tem enfoque no tratamento clinico de pessoas idosas. Nesse contexto segundo Silva \& Falcão (2014), apresentam o cuidador como parte da equipe de profissionais de saúde e que deveria ter entendimento em diversas áreas da saúde, sendo o foco principal o processo de envelhecimento e na melhoria do trabalho em equipe. Fontes et al. (2021) complementa atribuindo parte da qualidade de vida do idoso a harmonia do cuidado desenvolvido pelo cuidador.

O ofício de cuidador é ratificado como ocupação na Classificação Brasileira de Ocupações (CBO) do Ministério do Trabalho. Salienta-se que a CBO apenas classifica e não regulamenta as profissões. Contudo está em tramitação o Projeto de 
Lei $\mathrm{n}^{\circ} 76$ de 2020 que prevê requisitos legais para regulamentação do exercício da profissão de cuidador, dispondo a proposta que o cuidador de pessoas idosas, de crianças e pessoas com deficiência ou doença rara, se enquadre nas seguintes exigências: ter 18 anos completos, ter concluído pelo menos o ensino fundamental ou equivalente, ter curso de qualificação para cuidador, não apresentar antecedentes criminais e atestar não ter problemas físicos e psíquicos.

Os cuidadores são responsáveis por assistir o idoso de forma integral atendendo suas demandas de cuidados básicos, dentre eles o de higienização que compreende o banho no leito. Esse procedimento é realizado em pacientes que perderam a autonomia, capacidade motora, tátil, pessoas que vivem acamadas, inclusive os idosos, que são incapazes de realizar o autocuidado, seja nos casos de lesões ou tratamentos que impeçam ou possa limitar a funcionalidade dos membros inferiores, ou em determinados tratamentos em que o repouso absoluto é exigido (Nepomuceno et al., 2014).

Durante a aplicação do banho no leito, praticado nos hospitais e nas ILPIs é notável no dia-a-dia que há carência de uma oferta de qualidade em relação a higiene das pessoas confinadas no leito, ficando assim a mercê do chamado "banho de toalha". Contudo, esse tipo de procedimento não assegura a retirada da sujidade e faz com que os profissionais fiquem transitando com bacias de água previamente aquecidas, perdendo tempo e não atendendo as principais necessidades da higienização (Albuquerque, 2013). Sob esta perspectiva o estudo objetivou descrever o conhecimento dos cuidadores, quanto a realização do banho no leito em uma instituição de longa permanência para idosos.

\section{Metodologia}

A presente pesquisa caracterizou-se como um estudo descritivo exploratório, com abordagem qualitativa. Foi realizada em uma ILPI filantrópica, localizada na zona urbana de um município cearense e contou com a participação de cinco cuidadores que prestavam serviços a instituição. Salienta-se, que apesar da amostra do estudo ser composta por um número relativamente pequeno, deve-se levar em consideração que os estudos de cunho qualitativo, priorizam a qualidade do conteúdo, ou seja, a riqueza das entrevistas, ao invés, do quantitativo de participantes.

Para participação na pesquisa os cuidadores deveriam se enquadrar nos seguintes critérios de inclusão: ter idade igual ou superior a 18 anos, prestar assistência ao idoso diariamente, especificamente realizar o banho no leito. Para exclusão do cuidador na amostra final, utilizou-se como critério: profissionais que estivessem de férias, licença ou atestado médico do trabalho durante o período de coleta.

Os dados foram coletados no mês de março do ano de 2017, por meio de entrevista semi-estruturada guiada por um roteiro que continha perguntas que respondem o objetivo traçado, elaborado em duas partes, a primeira parte abordando dados sociodemográficos e a segunda parte contendo dados direcionados a pesquisa, ambas constituídas por questões subjetivas, para facilitar a coleta, organização e análise dos dados. As entrevistas foram gravadas com o auxílio de um gravador e sequencialmente foram transcritas na íntegra.

O primeiro contato antes de iniciar a coleta de dados foi feita com a coordenadora da ILPI, que recebeu a pesquisadora e disponibilizou os horários de trabalho dos cuidadores. Dessa forma a pesquisadora se deslocou até a ILPI no dia de plantão de cada cuidador, fez abordagem com cada profissional explicando sobre a pesquisa e buscando a participação dos mesmos. As entrevistas foram realizadas em uma sala reservada, durante o horário de menos movimentação para cuidador, tendo uma duração média de 10 minutos.

A interpretação dos dados foi realizada com base na análise de conteúdo, cujo desenvolvimento deu-se em três fases. Na primeira delas, denominada pré-análise, os pesquisadores realizaram a leitura flutuante do material. Na segunda etapa, conceituada como exploração do material, definiram as unidades de registro desinteirando-as do corpus textual e classificandoas com emprego da técnica de calorimetria, em sequencia foram agrupadas em categorias empíricas. Por fim, na terceira e 
última fase, definida como tratamento dos resultados, realizou-se a interpretação e discussão dos achados na pesquisa à luz da literatura cientifica (Minayo, 2014).

O estudo respeitou todas as recomendações formais advindas da Resolução no 466/2012 e sua complementar 510/16 do Conselho Nacional de Saúde (CNS) referente a estudos envolvendo seres humanos. Também teve aprovação do Comitê de Ética em Pesquisa da Faculdade de Juazeiro do Norte com o parecer de n ${ }^{\circ}$ 1.974.426 e CAAE n ${ }^{\circ}$ 64964017.0.0000.5624. Os participantes foram informados dos objetivos da pesquisa e de seus benefícios, manifestando o aceite em participar desta, por meio da assinatura do Termo de Consentimento Livre e Esclarecido. Para assegurar seu anonimato foram atribuídos pseudônimos aos participantes (C1, C2, C3 ...).

\section{Resultados e Discussão}

Com base nas características sociodemográficas dos cinco cuidadores, a faixa etária variou de 23 a 39 anos, observouse prevalência do sexo feminino, representando um total de três cuidadoras. Quanto ao grau de formação, dois possuem ensino médio completo, um ensino fundamental completo, dois possuem ensino superior completo, salienta-se que todos possuíam curso que os habilitassem como cuidadores de idosos. No tocante tempo de trabalho na instituição foi variável, sendo de 15 dias a dois anos, entretanto quatro cuidadores já tinham prestado serviços a idoso antes de ingressar na instituição.

Com relação a análise do conteúdo emergiram três categorias que foram originadas a partir das respostas dos participantes, foram elas: 1 . O conhecimento científico como base para realização do trabalho do cuidador; 2 . A técnica de banho no leito e materiais utilizados pelos cuidadores; 3 . Banho proporcionando conforto e bem estar ao cliente.

A primeira categoria, denominada "O conhecimento científico como base para o trabalho do cuidador", reflete a percepção dos cuidadores sobre o conhecimento científico para proceder com seu trabalho, principalmente com relação a técnica do banho no leito, visto que proporciona segurança ao cuidador, além de conforto a quem o recebe, e quando esse cuidado é direcionado às pessoas idosas esse saber é fundamental, por se tratar de um grupo frágil com necessidades de cuidados elaborados e direcionados para a prestação do bem-estar.

O conhecimento científico é a base para ser feito o banho, como ele deve ser realizado, todas as técnicas e o passo a passo do procedimento. Além de proporcionar segurança a quem está realizando, pois, o cuidador tendo conhecimento ele não irá realiza-lo de qualquer forma (C3).

O cuidador que tem conhecimento cientifico proporciona segurança, ele sabe que não vai está realizando algo de maneira inadequada e sem falar que o banho é um procedimento simples, mais se não tiver um pouco de conhecimento você pode causar dano ao paciente (C2).

O banho no leito, apresenta-se como muito mais que um cuidado básico de higiene com a finalidade de deixar a pessoa limpa, exigindo conhecimentos específicos para sua realização. Afigura-se como um momento essencial na prestação e orientação dos cuidados, proporcionando o desenvolvimento da relação e interação entre cuidador e pessoa, gerando uma oportunidade de partilha de saberes (Lôbo \& Saraiva, 2017; Oliveira et al., 2020). Desse modo, é necessário que as instituições que prestam cuidados direcionados a melhorar a qualidade de vida dos idosos, sigam o que está disposto em lei e contratem cuidadores com uma formação mínima, incluindo cursos voltados a cuidados com idosos, e se possível cuidadores que tenham graduação em áreas da saúde.

Observou-se também falta de conhecimento dos entrevistados sobre as lesões por pressão que podem ser ocasionadas ao idoso durante o banho, sendo primordial para o cuidador conhecer a conduta que deve ser realizada para manter a 
integridade da pele do cliente. Com essa mesma percepção Prado et al. (2017) afirmam que o momento do banho é uma ocasião propícia para redução da incidência de infecções e de lesão por pressão, melhorando a qualidade de vida do cliente.

Entretanto quando o banho no leito é realizado com emprego da técnica incorreta efeitos colaterais no paciente como perturbação do seu bem-estar e maior risco de infecções são iminentes, além disso, a literatura cientifica versa acerca da necessidade de atentar-se a pacientes que apresentam comorbidades cardíacas ou respiratórias, pois durante esse procedimento há uma desestabilização na saturação de oxigênio corporal que voltará ao normal após 15 minutos, ademais um estudo realizado com pacientes oncológicos identificou uma queda significativa na glicemia destes pacientes, após a realização do banho no leito. Percebe-se, então, a importância do cuidador com um preparo cientifico adequado para identificar e lidar com possíveis complicações, benefícios e aplicação de técnicas corretas (Paula et al., 2017b; Oliveira \& Lima, 2020 ).

Para maior êxito do procedimento recomenda-se ao profissional que ao realizá-lo valorize a rotina de arrumação normal do cliente, horário do dia em que se procede rotineiramente a higiene, priorizando o cuidado de acordo com as preferências do cliente. Quanto a duração e a maneira empregada para dar o banho dependem das capacidades físicas do cliente, dos problemas de saúde e do grau de higiene necessário (Potter \& Perry 2018).

Em síntese, a presente categoria clarifica a relevância do saber científico no trabalho com cuidador dando enfoque no procedimento de banho no leito, nesse mesmo sentido Costa et al. (2018) referem que a complexidade desse cuidado associada a condição de vulnerabilidade do cliente exige para um bom desempenho do profissional o embasamento cientifico.

Na segunda categoria, nomeada "A técnica de banho no leito e materiais utilizados pelos cuidadores", emergiu a compreensão de que os cuidadores seguem um passo a passo para realizar esse procedimento, inicialmente conversando com o cliente e explicando-o o procedimento, posteriormente realizando o banho propriamente dito, no sentido cefalocaudal, sempre iniciando do lado com menor sujidade para o lado com maior sujidade, além de tudo, sempre preservando a privacidade do cliente.

Primeiro, tudo que eu vou fazer com a paciente eu explico a ela o que está sendo realizado e o porquê que está sendo realizado [...] começo sempre de cima para baixo, do lado menos infectado para o mais infectado, sempre começo do meu lado oposto [...] e tudo que utilizo já vou desprezando, nada é reutilizado e sempre mantenho a integridade do paciente, através de biombos, no caso do abrigo fecho as portas da ala e após fazer essa higienização, manter ela bem aquecida, coberta, vestir a roupa fazer tudo começando pela face ai você vai descendo para membros superiores, depois tórax e membros inferiores para limpar depois a região da vagina ou pênis e ânus, primeiro a vagina depois o ânus por ele ser o mais contaminado e depois tentar dar um conforto melhor, penteando o cabelo e passando colônia $(\mathrm{C} 1)$.

Nesse sentido Moller e Magalhães (2015), apontam que o banho no leito deve ser realizado de maneira responsável e ética, deve ser seguido o passo a passo da maneira como é recomendado, proporcionando ao cuidador segurança. Todo banho no leito quando realizado, tem que seguir um roteiro, para que assim o procedimento tenha êxito e não ocorra erro na sua execução.

Dentre os pontos positivos identificados na fala do cuidador destaca-se a privacidade e o respeito com o corpo desnudado. $\mathrm{O}$ banho no leito gera intimidade entre profissional e cliente e causa desconforto de ambas as partes, o que muitas vezes faz com que esse tipo de cuidado seja realizado em silêncio. Isso leva a discutir outro aspecto da fala do cuidador: a comunicação que o mesmo tem com o idoso. O diálogo é essencial em todos os momentos do banho, pois deixa o paciente mais tranquilo e facilita a realização do banho (Figueiredo et al., 2006; Martins, 2009). 
Um ponto importante não foi referido pelo cuidador, trata-se dos aspectos de segurança do idoso, Potter \& Perry (2018) relatam a necessidade de manter a segurança com as grades laterais elevadas quando estiver distante do leito no caso de pacientes dependentes ou inconscientes.

Ao indagar os cuidadores sobre quais materiais são utilizados no banho no leito, recebeu destaque a importância de se ter o material para higienização, como: xampu, sabonete, hidratante e algodão.

No caso de abrigo é mais complicado, porque nem sempre tem tudo, mais eu sempre prezo ter algodão, água, sabonete líquido, asseptol e toalha. Sempre separar o material antes e colocar ao seu alcance, é interessante que você se puder ter auxilio de alguém durante o banho, eu sempre estou com gazes, xampu e creme, não usamos todos os dias e sim em dias intercalados, utilizo também cubas e é basicamente isso (C2).

Depreende-se da fala do cuidador dois aspectos relevantes como o conhecimento dos materiais usados para o banho no leito e as dificuldades para sua aquisição. Evidencia-se, assim que esse profissional apresenta conhecimento de parte dos materiais que devem ser utilizados para realizar o banho no leito que de acordo com Pereira \& Zorita (2013) são toalha de rosto, cuba-rim, gaze, carrinho de banho, biombo, hamper com saco plástico, jarra com água morna, fralda geriátrica, materiais de higiene pessoal (xampu, condicionador, pente, toalha de banho, desodorante, hidratante) e os equipamentos de proteção individual (EPIs), a saber máscara, luvas de procedimento e avental. No entanto, os cuidadores não citam em suas falas os EPIs, contudo esses equipamentos são essenciais para segurança do cuidador e do idoso, Moller \& Magalhães (2015) enfatizam a extrema necessidade de utilizar esses equipamentos e associá-los técnica asséptica correta para que o banho seja efetivo e não prejudicial.

Ainda sobre os produtos utilizados, ressalta-se que devido às condições acarretadas pela idade, a pele do idoso tornase mais frágil, assim durante o banho, para evitar maiores danos deve-se utilizar sabão neutro e evitar produtos que ressecam a pele, a exemplo os produtos que tenham teor alcoólico, como é o caso do asseptol mencionado pelo cuidador. É recomendado o uso de cremes e desencorajado o uso de talcos, embora este último seja preferido pelos idosos podem prejudicar a pele (Paula et al., 2017b).

Mesmo com as recomendações de utilização de todos os materiais acima expostos, existem algumas ILPIs que passam por dificuldades financeiras, dessa forma nem todos esses produtos são disponibilizados, o que pode prejudicar a qualidade do procedimento. Esse achado também foi encontrado em um estudo que trata do perfil nutricional de idosos de ILPIs, assim mostra que as instituições de cunho filantrópico apresentam carências em relação aos recursos financeiros (Camargos et al., 2015).

É pertinente frisar, nessa discussão, que apesar do cuidador referir a importância de outra pessoa para prestar auxilio no momento do banho, a maiorias das vezes estão sozinhos com o idoso, o que gera, além de tudo, maior esforço físico e sobrecarga do cuidador. Conforme Moller \& Magalhães (2015) e Bastos et al. (2019) esse é um disparador de risco para segurança dos profissionais, desse modo recomendam que o procedimento seja realizado por mais de um profissional.

$\mathrm{Na}$ terceira e última categoria intitulada "O banho proporcionando conforto e bem estar ao cliente", os cuidadores apontam para o banho como meio para favorecer o bem-estar a saúde do idoso, na maioria das vezes melhora a aparência, e proporciona conforto físico.

Banho é saúde, então assim você quando está com um idoso que ele toma banho duas vezes ao dia que você mantém as unhas cortadas e faz a higienização correta como a da vagina, que a gente sabe que senão for feita corretamente vai adquirir corrimento que gera outras doenças. Então o banho proporciona conforto bem-estar (C1). 


\section{Melhora o sono, proporciona conforto e sensação de limpeza (C4).}

O entendimento dos profissionais está de acordo com o estudo de Paulela (2016) que afirma que o banho no leito melhora o bem-estar e tem dentre suas finalidades proporcionar a integridade da pele, defesa contra infecções, reduzir odores corporais e melhorar a autoimagem. As propriedades terapêuticas do banho sempre foram reconhecidas ao longo da história, no entanto, o banho passou a ser defendido pelo seu potencial higienizador da pele, pela estimulação sanguínea, pelo alívio do desconforto e relaxamento muscular, proporcionando a quem o recebe uma sensação de conforto, sensação de limpeza e melhora a autoestima do idoso (Backes et al., 2017).

Dias et al. (2016) referem ao banho no leito como um cuidado terapêutico, entende que este deve ser prestado por pessoas que tenham sensibilidade e que se preocupam com o boa disponibilidade e satisfação do paciente, a fim de que este momento se torne uma experiência agradável para todos os envolvidos, contribuindo para o alcance da integralidade do cuidado.

Assim, o banho não deve ser visto como uma simples técnica que apenas tem que seguir o passo a passo e com a finalidade de deixar a pessoa limpa. É necessário que os profissionais que o realizam tenham a intenção de cuidar, pois quando quem executa essa técnica com destreza, paciência e afeto, pode transformar cada ato por mais simples que ele seja, para aquela pessoa que o recebe, não é apenas um banho e sim um gesto de cuidado, que esta sendo realizado para proporcionar a saúde e bem-estar (Martins, 2009). Com isso é necessário que o cuidador ao realizar o banho no leito, tenha simpatia e ternura com o indivíduo que esta recebendo o cuidado, sempre proporcionando conforto e contentamento.

\section{Considerações Finais}

Diante do contexto, é notável que a realização do banho no leito é de grande relevância, pois a higiene corporal é uma necessidade humana básica imprescindível para a condição de vida saudável. Assim, o cuidador deve estar capacitado e ter conhecimento para desenvolver o procedimento com habilidade e segurança, pois aplicação de técnicas corretas torna o procedimento seguro e eficaz.

Percebeu-se que os cuidadores participantes desse estudo têm conhecimento considerável acerca da técnica de banho no leito, entretanto ainda existem lacunas em seus conhecimentos que aliadas às barreiras como a carência de alguns materiais para higienização, prejudica a qualidade do procedimento. A identificação de problemas de assistência em saúde e a busca conjunta por sua resolução faz parte do processo de educação permanente, podendo então ser uma estratégia para os cuidadores melhorarem o atendimento ao idoso não apenas no que tange ao banho no leito, mas em todo o processo de cuidado.

Apesar dos entraves, os cuidadores prestam assistência aos idosos de uma forma humanizada, sempre proporcionando o bem-estar, fazendo que por meio do banho eles se sintam mais confortáveis. Por fim, sugere-se a necessidade de estudos mais amplos sobre a temática envolvendo outros cenários, como ILPIs privadas.

\section{Referências}

Albuquerque, K. F. (2013). Tecnologia para banho no leito de idosos e usuários acamados: uma contribuição para o cuidar humanizado, Tese de doutorado, Universidade Federal do Rio Grande do Norte, Natal, Brasil.

Backes, D. S., Gomes, C. A., Pereira, S. B., Teles, N. F., \& Backes, M. T. S. (2017). Banheira portátil: tecnologia para o banho de leito em pacientes acamados. Revista Brasileira de Enfermagem, 70 (2), 364-369.

Bastos, S. R. B., Gonçalves, F. A. F., Bueno, B.R.M., Silva, G.S., Ribeiro, K. R. A., Brasil, V. V.(2019). Banho no Leito: Cuidados Omitidos pela Equipe de Enfermagem. Rev Fund Care Online, 11(3): 627-633.

Brasil. (2006). Ministério da Saúde. Secretaria de Atenção à Saúde. Departamento de Atenção Básica. Envelhecimento e Saúde da Pessoa Idosa. Cadernos de Atenção Básica. Brasília, DF: Ministério da Saúde. 
Brasil. (2002). Portaria n 397, de 10 de dezembro de 2002. Institui a Classificação Brasileira de Ocupações. Ministério do Trabalho e Emprego.

Camarano, A. A., \& Kanso, S. (2010). As instituições de longa permanência para idosos no Brasil. Revista Brasileira de Estudos de População, 27 (1), 232235 .

Camargos, M. C. S., Nascimento, G. W. C., Nascimento, D. I. C., \& Machado C. J. (2015). Aspectos relacionados à alimentação em Instituições de Longa Permanência para Idosos em Minas Gerais. Cad. saúde colet., 23(1), 38-43.

Costa, M. C. N. S. \& Mercadante, E. F. (2013). O Idoso residente em ILPI (Instituição de Longa Permanência do Idoso) e o que isso representa para o sujeito idoso. Revista Kairós Gerontologia, 16(2), 209-222.

Costa, G. S., Souza, C. C. de, Diaz, F. B. B. S., Toledo, L. V. (2018). Banho no leito em cuidados críticos: uma revisão integrativa. Rev baiana enferm, 33, e20483.

Dias, J. A. A, Souza, D. M., Bruno, D. S. A., Andrade, I. S., \& Nery, P. I. G. (2016). O banho no leito na óptica de estudantes de graduação em enfermagem. Revista de Pesquisa: cuidado é fundamental Online, 8(4), 5087-5094.

Figueiredo, N. M. A. de, Carvalho, V. de, \& Tyrrell, M. A. R. (2006). (Re)lembrando Elvira de Felic: gestos e falas de enfermeiras sobre o banho no leito, uma técnica/tecnologia de enfermagem. Escola Anna Nery, 10(1), 18-28.

Fontes, P. C., Monteiro, E. A., Oliveira, A. B. C., Oliveira, A. K. M., Moreira, M. A. S. P., Farias, I. A. P., \& Medeiros, R. A. de. (2021). Habitação e qualidade de vida de idosos: revisão integrativa. Research, Society and Development, 10 (2), e8910212277.

Lôbo, C. R., \& Saraiva, T. L. A. (2017). Importância do procedimento banho no leito para atendimento em enfermagem. Revista Recien, 7(20), 82-90.

Martins, S. A. I. (2009) O banho no leito em contexto de internamento hospitalar. vivências de pessoas idosas, Dissertação de mestrado, Instituto de Ciências Biomédicas Abel Salazar, Universidade do Porto, Portugal.

Minayo, M. C. S. (2014). O desafio do conhecimento: pesquisa qualitativa em saúde. São Paulo, SP: Hucitec.

Moller, G. \& Magalhães, A. M. M de (2015). Banho no leito: carga de trabalho da equipe de enfermagem e segurança do paciente. Texto Contexto Enferm., 24(4), 1044-1052.

Nepomuceno, B. C., Campos, B. C., Simões, I. A. R., \& Vitorino, L. M. (2014). Banho No Leito: o Discurso do Sujeito Coletivo de Pacientes Hospitalizados. Revista Ciências em Saúde, 4(1).

Oliveira, A. P. de, \& Lima, D. V. M. de. (2020). Glycemic repercussions of bed bath of critically ill cancer patients: clinical trial. Research, Society and Development, $9(8)$, e08985353.

Oliveira, S. M. de, Costa, K. N. de F., Santos, K. F. O dos, Oliveira, J. dos S., Pereira, M. A., \& Fernandes, M. das G. M. (2020). Necessidade de conforto percepcionada por idosos hospitalizados: uma análise à luz da teoria de Kolcaba. Revista Brasileira de Enfermagem, 73(3), e20190501.

Organização das Nações Unidas. A ONU e as pessoas idosas. Acesso: https://nacoesunidas.org/acao/pessoas-idosas/.

Paula, R. T. de, Sousa M. E. F. P., Reis, T. M., Santos, L. A. C., Resende, M. A., \& Souza, G. (2017a). A atuação do enfermeiro diante a depressão em idosos institucionalizados: subsídios de prevenção. Revista Eletrônica Acervo Saúde. 11, 1053-1060.

Paula, M. F. C, Santos, E. R., Silva, M. R da, \& Bergamasco, E. C. (2017b) Semiotécnica: fundamentos para prática assistencial em enfermagem. Rio de Janeiro, RJ: Elsevier.

Paulela, D. C. (2016). Banho no leito convencional e descartável: estudo microbiológico e de custo, Dissertação de Mestrado, Faculdade de Medicina, Universidade Estadual Paulista, Botucatu, São Paulo, Brasil.

Pereira, S. C., \& Zorita, L. A. (2013). Procedimento Operacional Padrão: Banho de aspersão com auxílio. UNIFESP.

Poltronieri, B. C., Souza, E. R., Ribeiro, A. P. Violência e direito ao cuidado nas políticas públicas sobre instituições de longa permanência para idosos. Interface - Comunicação, Saúde, Educação. 23, e180124.

Potter, P.A, \& Perry, A. G. (2018). Fundamentos de enfermagem. GEN Guanabara Koogan.

Prado, A. R. A., Ramos, R. L., Ribeiro, O. M. P. L, Figueiredo, N. M. A. de, Martins, M. M., \& Machado, W. C. A. (2017). Bath for dependent patients: theorizing aspects of nursing care in rehabilitation. Revista Brasileira de Enfermagem, 70(6), 1337-1342.

Silva, M. P., \& Falcão, D. V. S. (2014). Cuidar de Idosos numa ILPI na Perspectiva de Cuidadoras Formais. Revista Kairós - Gerontologia, $17(3)$, 111-131. 